\title{
Soft Renal Calyx Calculi under Ureter Mirror Treatment
}

\author{
Fan-ping MENG ${ }^{1, a}$, Yan $\mathrm{HE}^{2}$, Di ZOU² and Ji GAO ${ }^{1, b, *}$ \\ ${ }^{1}$ Department of Urology, China-Japan Union Hospital, Jilin University, \\ Changchun, Jilin, China \\ ${ }^{2}$ Department of Hepatology, Jilin Academy of Traditional Chinese Medicine, \\ Changchun, Jilin, China \\ a947410581@qq.com, b1217760@qq.com \\ ${ }^{*}$ Corresponding author
}

Keywords: Soft Ureter Mirror, Renal Calyx Calculi.

\begin{abstract}
Objective: to study the electronic soft ureter mirror joint qin laser lithotripsy in the treatment of renal calyx calculi under safety and effectiveness.Methods: our hospital in March 2016 - March 2017 electronic soft ureter mirror qin laser lithotripsy under 40 patients under the treatment of renal calyx calculi, stone diameter $1.02 .0 \mathrm{~cm}$, $1.4 \mathrm{~cm}$.Results: 38 cases were soft issue of successful completion of the ureter mirror lithotripsy, 2 cases with ureteral lumen narrow, soft ureter mirror cannot be upward, indwelling double $\mathrm{J}$ tube line again after 2 weeks of ureteral soft lens operation is a success.Operation time 30-95 min, the average $55 \mathrm{~min}$, were not serious perioperative complications, postoperative 1 to 2 months follow-up, no urinary calculi residual or residual stone diameter $<3 \mathrm{~mm}$. Conclusion: electronic soft ureter mirror digital image clarity, vision amplification, and qin laser under the combination therapy of renal calyx calculi is safe and effective, and is worth popularizing in clinical use.
\end{abstract}

\section{Introduction}

Urinary calculi in $40 \%-50 \%$, kidney stones with renal calyx calculi under is about $36 \%[1]$.The calyx calculi because of its unique anatomical structures, etc., extracorporeal shock wave Lithotripsy (extracorporeai shock wave Lithotripsy ESWL) after the stone clearance rate is low, bring considerable difficulties to clinical treatment, with the continuous development of soft ureter mirror, especially the emergence and development of electronic soft ureter mirror, for the treatment of renal calyx calculi under provides a good method.I division in March, 2016 - March 2017 using electronic soft ureter mirror joint qin laser lithotripsy under the treatment of renal calyx calculi 40 cases, curative effect is satisfied, the coverage is as follows:

\section{Data and Methods}

This group of 40 cases, 25 cases of male, female, 15 cases, 18-76 years old, 46 years old on average. 29 cases because of the discomfort, the back pain symptoms such as hematuria, 3 days - 15 months duration. 8 cases of course $<3$ months, 8 cases of 3-6 months, > 14 cases of 6 to 12 months, 1-3 years 3 cases;More than 7 cases without clinical symptoms, medical examination found: both by ultrasound, intravenous urinary tract imaging (April) and CT etc. Calyx calculi under inspection diagnosed with kidney stones, $1.02 .0 \mathrm{~cm}$ in diameter, an average of $1.4 \mathrm{~cm} .34$ patients who underwent ESWL treatment, the effect not beautiful.Selection criteria: renal calyx calculi, diameter $<2$ $\mathrm{cm}$. 
1.2 General anesthesia, cut stone: F8.0/9.8 into the bladder, ureteral hard mirror to the side of ureteral into the thread, the thread will be guided by hard ureter mirror into the side of the ureter, on to the renal pelvis and ureter expansion, and retained the godet, exit the ureter mirror, along the thread of ureteral soft lens outer sheath, pulling thread and electronic soft ureter mirror STROZ (Germany) look straight down along the outer sheath into the renal pelvis, soft lens into the renal pelvis and enter the calyx, looking for stone, and then soft ureter mirror work placement channels with 200 microns qin laser optical fiber, optical fiber connection PowerSuite100 W qin laser lithotriptor (Lumenis medical laser, medical people power $0.81 .5 \mathrm{~J}, 10-20 \mathrm{hz}, 10$ to $30 \mathrm{~W}$ ) fiber out soft ureter mirror front $0.5 \mathrm{~cm}$, crushed stones. Use infusion pump or assistant syringe injection at the same time ensure clear vision. If the stone position is partial, gravel is difficult, you can use the set of stone on top place calculi after trapping in rubble after the renal pelvis, stone smash to diameter $<3 \mathrm{~mm}$ pieces. For larger pieces of stone, the use of set of stone on top (American Cook) trapping stones from soft ureter mirror outer sheath after in vitro. Gravel satisfied, lien thread in the renal pelvis, withdraw soft ureter mirror, withdrawn from the outer sheath, along the thread indwelling double $\mathrm{J}$ tube

\section{The Results}

First phase of 38 cases were successfully complete ureteral soft lens lithotripsy, 2 cases with ureteral lumen narrow, electronic soft ureter mirror cannot be upward, indwelling double $\mathrm{J}$ tube line again after 2 weeks of ureteral soft lens operation is a success. Intraoperative, there was no serious complications, has not occurred ureter perforation, avulsion fracture, fever, bleeding complications, etc. Operation time 30-95 min, flat, 55 min.2-5 d after hospital discharge, an average of $4 \mathrm{~d}$. After 2 to 4 weeks after remoal of double $\mathrm{J}$ tube. 1 to 2 months after B ultrasonic and X-ray check abdominal plain film (KUB), without urinary tract stones residual or residual stone diameter $<3 \mathrm{~mm}$.

\section{Discussion}

With the continuous development of orifice translumenal endoscopic technology, the continuous improvement of the crusher and stone tools, revolutionary changes have taken place in the treatment of urinary calculi, traditional open surgery has gradually been represented by orifice translumenal endoscopic minimally invasive treatment [2].Renal calyx calculi under due to its anatomical particularity, treatment is difficult.At present, the treatments with ESWL, percutaneous nephroscope technology (PCNL), soft ureter mirror, etc. ESWL treatment for renal calyx calculi under under the light of the anatomical structure, size, composition, number, and stones, etc [3].Although the position row stone can help, but the overall stone rate is low, at just 37\%.The curative effect is poorer. When the stone $>1 \mathrm{~cm}$ in diameter, with the increase of stone diameter, stone clearance rate gradually decreases after ESWL. PCNL curative effect, but need from renal parenchyma drilling into the renal pelvis, trauma is bigger, have more severe complications such as bleeding, infections, etc., can appear sometimes renal vascular embolization, nephrectomy, septic shock and even death [4]. The procedure used to treat larger stones, such as stone diameter $>2 \mathrm{~cm}$ below calyx calculi. Retrograde ureteral soft treatment of kidney damage between PCNL and ESWL. Over the past 20 years, as the medical engineering technology, electronic technology, the rapid development of material technology, increasingly perfect soft ureter mirror and form a complete set of gravel equipment, for the treatment of kidney stones has opened up a 
new way to safe, effective and minimally invasive. Especially the emergence of electronic soft ureter mirror, make the image magnification to further increase. Observation clarity greatly increase, the operation more flexible, than in the past ordinary optical soft lens have a qualitative leap. With the development of qin laser technology, 200 micron ultra soft optical fiber than ordinary optical fiber is more soft, soft lens to affect greatly reduced. Of retrograde ureteral soft lithotripsy and holmium laser for the treatment of kidney stone has obtained the good effect. For renal calyx calculi under can also use the set of stones on top first place renal calyx calculi under rubble after the renal pelvis. Shvarts reports such as soft ureter mirror in the use of nickel titanium alloy stone basket, 200 micron and 360 microns laser optical fiber can cause maximum bending Angle decrease by $4.4 \%, 9.9 \%$ and $4.4 \%$, respectively, therefore, for the calyx calculi can use first set of stone basket gravel stones will drag after renal pelvis. Schuste reported using in situ and reset after using ureteral soft lens under the treatment of renal calyx calculi, $1-2 \mathrm{~cm}$ in diameter of renal calyx calculi under in situ group effective rate was $29 \%$, reset group was $100 \%, 71 \%, 94 \%$ of the total stone clearance rate. Using electronic soft ureter mirror under the rubble, also need to choose the appropriate qin laser lithotripsy power, general khin laser lithotripsy power of 0.8-1.0 J / 10-20 hz. High power laser beam refraction, could occur damage soft lens, yi also high energy loss optical fiber, generally USES the $<30 \mathrm{~W}$ to gravel, small power for hard stone adjustable high power to 30-40 W to rubble [5]. Gravel method basically has the following two kinds: one starts from the area surrounding the stone, crushed stones, using relatively small power stones crushed into $<3 \mathrm{~mm}$ pieces, to facilitate postoperative stone pieces out of the body; Two starts, stone central will be divided into larger stones pieces, and then the rubble, respectively. For larger residual calculi, can use a set of stone on top show the stone fragments and remove. Electronic soft ureter mirror line of calyx rubble stone extraction for need to pay attention to the following: (1) soft ureter mirror upside ureteral ureteroscopy direction to adjust, the ureteral lumen has always placed in central vision, ureteral lumen stenosis through mirror difficulties, avoid blindly violence on the mirror, can be placed first ureteral double-j tube expansion of ureteral lumen 1-2 weeks, and then again ureterorenoscopic realignment surgery.(2) for renal calyx calculi, can first use set stone on top set to pelvic, then to rubble.(3) using qin fiber laser 200 microns of gravel, pay attention to setting up the appropriate power and frequency, fiber out of rubble soft ureter mirror 5-10 mm, prevent damage soft ureter mirror.(4) $<3 \mathrm{~mm}$ stone fragments can pa ontaneously, $>3$ $\mathrm{mm}$ stone stone fragments can be used to set out on top in vitro.(5) is located in the calyx calculi fragments to the renal pelvis, it can use water to facilitate the back stone. 6 . The entire operation process especially for infectious stones, perfusion pressure is unfavorable and exorbitant, try to reduce the operation time, don't be too much emphasis on gravel extent, prolong operation time.

\section{Conclusions}

In short, soft ureter mirror qin renal calyx calculi under laser lithotripsy has the characteristics of minimally invasive, safe and efficient, especially with more excellent performance in the clinical applications of electronic soft ureter mirror, stone treatment success rate may be further improved. 


\section{References}

[1] Multi-session retrograde endoscopic lithotripsy of large renal calculi inobese patients. WHEAT JC, ROBERTS WW, WOLF JS JR, et al. The Canadian Journal of Urology. 2009

[2] Flexible ureteroscopy and holmium-Yag laser: material and technique. Saidi A, Combes F, Delaporte V, et al. Progress en Urologie . 2006

[3] Characterization of intrapelvic pressure during ureteropyeloscopy with ureteral access sheaths [J]. Jamil Rehman, Manoj Monga, Jaime Landman, David I Lee,Tamer Felfela, Marius C Conradie, Rajamahanty Srinivas, Chandru P Sundaram, Ralph V Clayman. Urology. 2003 (4)

[4] Flexible ureterorenoscopy versus miniaturized PNL for solitary renal calculi of 10-30 mm size. Knoll T, Jessen JP, Honeck P, et al. World Journal of Urology . 2011

[5] Flexible Ureteroscopy and Laser Lithotripsy for Stones $>2 \mathrm{~cm}$ : A Systematic

Review and Meta-Analysis. Omar M. Aboumarzouk, Manoj Monga, Slawomir G. Kata. Journal of Endourology. 2012 